\title{
THE EFFECT OF STRAIN SOFTENING IN THE MATRIX MATERIAL DURING VOID GROWTH
}

\author{
Guo Chen Li† \\ Institute of Mechanics, Academia Sinica, Beijing, China \\ and \\ I. C. Howard \\ Department of the Theory of Materials, Sheffield University, \\ Mappin Street, Sheffield, S1 3JD, U.K.
}

(Received 24 June 1982; in revised form 1 September 1982)

\section{ABSTRACT}

A FINITE element analysis has been employed to investigate the growth of an initially spherical void embedded in a cylinder of elastic-plastic material. The boundary displacement of this cylindrical cell is regulated by the value of a parameter $\alpha$ which controls the radial shrinkage of the cell as it elongates. A large strain analysis was used and results for both strain hardening and strain softening (after an appropriate amount of hardening has taken place) have been obtained. The effects of different mean tensile stresses, equivalent strains and initial void volume fractions have also been included. The numcrical work shows relationships between the mechanical and geometrical variables that may favour ductile fracture by void coalescence or by shear decohesion.

\section{NOTATION}

$$
\begin{aligned}
& \tau^{i j} \quad \text { Kirchhoff stress tensor } \\
& \sigma^{i j} \quad \text { true stress tensor } \\
& V_{i} \quad \text { velocity of displacement vector, i.e. } V_{i}=\dot{U}_{i} \\
& \dot{F}^{i} \quad \text { load rate vector } \\
& g^{i j}, g_{i j} \quad \text { metric tensor } \\
& \frac{\mathscr{D}}{\mathscr{D}^{t}}(\text { ) Jaumann derivative } \\
& l_{j} \quad \text { covariant derivative } \\
& S^{i j} \quad \text { deviatoric stress }\left(=\sigma^{i j}-\frac{1}{3} g^{i j} g_{k l} \sigma^{k l}\right) \\
& \sigma_{\mathrm{e}} \quad \text { equivalent stress }\left[=\left(\frac{3}{2} g_{i k} g_{j l} S^{i j} S^{k l}\right)^{1 / 2}\right] \\
& \varepsilon_{\mathrm{e}} \quad \text { equivalent strain }\left(=\int_{0}^{t} D_{\mathrm{e}} \mathrm{d} t\right), \quad \varepsilon_{e}^{(\mathrm{p})}=\int_{0}^{t} D_{\mathrm{e}}^{(\mathrm{p})} \mathrm{d} t \\
& D_{\mathrm{e}}=\left(\frac{2}{3} d_{j}^{i} d_{i}^{j}\right)^{1 / 2}, \quad D_{\mathrm{e}}^{(\mathrm{p})}=\left(\frac{2}{3} d_{j}^{i(\mathrm{p})} d_{i}^{j(\mathrm{p})}\right)^{1 / 2} \\
& d_{j}^{i}=D_{j}^{i}-\frac{1}{3} \delta_{j}^{i} g^{k l} D_{k l}, \quad d_{j}^{i(\mathbf{p})}=D_{j}^{i(\mathbf{p})}-\frac{1}{3} \delta_{j}^{i} g^{k l} D_{k l}^{(\mathbf{p})}=D_{j}^{i(\mathbf{p})}
\end{aligned}
$$

† Work completed whilst visiting the Department of Mechanical Engineering, Sheffield University, U.K. 


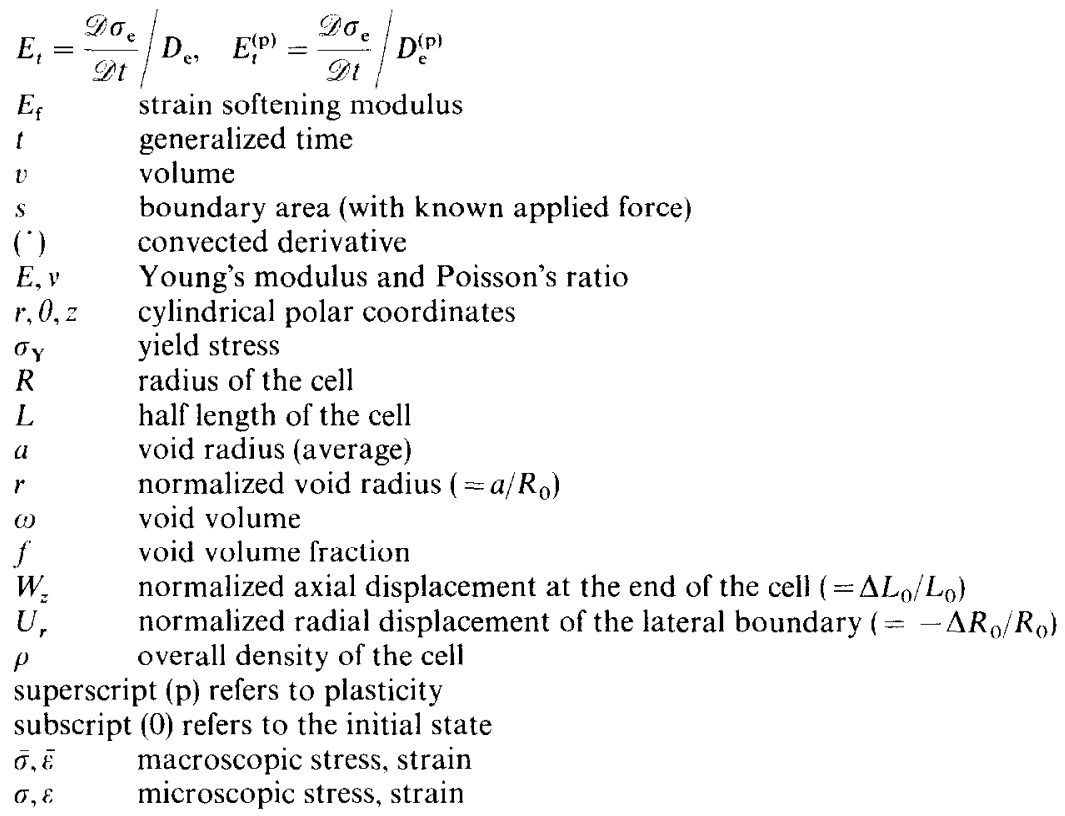

\section{INTRODUCTION}

THE THEORETICAL study of the micromechanics of ductile fracture began with the work of MCCLINTOCK (1968) on the enlargement of a cylindrical void in plastically deforming material and that of RICE and TRACEY (1969) on the growth of a spheroidal void. By analysing a single void in a spherical cell and a long circular cylindrical void in a cylindrical matrix GURSON (1977) developed approximate yield criteria and flow rules for porous (dilatant) ductile materials, showing the role of mean tensile stress in plastic yield and void growth. ANDERSSON (1977) used a finite-element method to examine the deformation of a circular cylindrical cell containing a spherical void. He estimated the point of fracture by supposing that it occurred when the growing void touched the cell wall, and he showed that the mean stress on the cell decreased as the void grew. Rice and Tracey (1969), Gurson (1977) and Andersson (1977) all assumed the matrix material to be non-hardening. MCCLINTOCK (1968) included the effects of strainhardening which cause an increase in fracture strain. NEEDLEMAN (1972) made use of a large plane-strain finite-element computation to analyse the growth of a cylindrical void in an elastic-plastic medium with different initial void volume fractions. His results showed the growth of the void and the plastic zone around it with increasing overall strain and also demonstrated that overall softening of the cell increased with the initial void fraction.

Recently, Hancock and Mackenzif (1976) and Hancock and Cowling (1980) working with low alloy, quenched and tempered steels, HANCOCK and BROWN (1983) working with plain carbon steels and the BEREMIN group (1981 a,b) working with A508 nuclear pressure vessel steel have all shown the importance of mean tensile stress in 
accelerating void growth rates and decreasing the overall fracture strain. The relationship between $\bar{\sigma}_{\mathrm{m}} / \bar{\sigma}_{\mathrm{e}}$ and $\bar{\varepsilon}_{\mathrm{e}}$ is exponentially decreasing. IRICIBAR, LE ROY and EMBURY (1980) and FISHER and GURLAND (1981) tested different spheroidized plain carbon steels and showed that the voids grew exponentially with strain.

The criteria that have been suggested for the initiation of ductile fracture fall into three main groups. In most of them there is a (perhaps implicit) recognition of the role of mean tensile stress in promoting rapid void growth and accelerating fracture, as the experimental work just mentioned requires.

\section{(1) Critical void size criteria}

BEREMIN (1981a) claims that the critical void size varies only slightly with mean tensile stress although, of course, the rate at which this critical size is approached will depend strongly on it. Both MCCLINTOCK (1968) and ANDERSSON (1977) suggest that fracture occurs when one of the semiaxes of the void reaches half of the cell size.

\section{(2) Damage function criteria}

Norris, REaugit, Moran and Quinonis (1978) assume that fracture occurs when the damage accumulated over a characteristic microstructure length becomes critical. The function of HANCOCK and MACKENZIE (1976) involves the ratio of mean and equivalent stress and the equivalent plastic strain as the main variables.

\section{(3) Material instability criteria}

When the overall softening of the cell due to void growth within it exceeds the strainhardening of the matrix the overall stress may attain a maximum and thereafter decrease as the deformation of the cell proceeds. Rousselier (1981) took this limit point on the stress-strain curve as the point of material instability. Softening also causes the overall density of material to decrease rapidly. Making use of these features, together with a strain localization technique in his finite-element mesh, he simulated stable crack growth in a three-point bend specimen. He used a damage parameter in his analyses as an internal variable of the material with the parameter being a function of the density and the initial void volume fraction.

The work reported in this paper introduces hoth material strain hardening and strain softening, after a suitable amount of plastic deformation, into a simple model of void growth. The model has the same geometrical form as that used by ANDERSSON (1977). But, instcad of having the lateral cylindrical boundary fixed, different amounts of radial contraction are assigned; the resulting axial and radial loading simulates different stress states. The large strain finite-element formulation of McMeEKING and RICE (1975) was used for the numerical work.

The effects on void growth of different mean stresses, equivalent strains and initial void volume fractions have been investigated. By allowing the matrix to harden indefinitely the achievable levels of stress within the cell at the point of its instability are unbelie'ably large. This effect is alleviated by allowing the matrix material to soften after a certain critical stress $\sigma_{\mathrm{c}}$ (which depends both on mean tensile stress and 


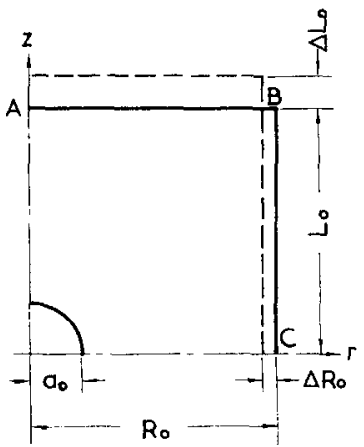

FIG. 1. A quadrant of the axisymmetric unit cell used to model the growth of a void in elastic - plastic material.

equivalent strain) has been reached. The resulting instabilities are similar to those of Rousselier (1981) who used a damage function but the more straightforward mechanical interpretation of our work may allow an easier connection between the macro- and micro-response $t$ of the material and provide a better tool for the analysis of experiment. A further advantage of the use of softening lies in the fact that the pattern of softening in the cell as deformation proceeds depends strongly on the mechanical and geometrical parameters of control, and different patterns can be seen to favour one or other of the fracture mechanisms of void coalescence or shear decohesion. A study of these patterns then suggests which combination of the control parameters favours which fracture mechanism.

\section{The Model of Void Growth and the Computational Method}

We have analysed the deformation of a circular cylindrical cell with a spherical void at the centre; a quadrant of its section is shown in Fig. 1. The reaction of the body containing this cell is represented by the value of a parameter $\alpha$ which controls the radial shrinkage of the cell as it elongates. Specifically

$$
\frac{\Delta U_{r}}{R / R_{0}}=-\alpha \frac{\Delta W_{z}}{L / L_{0}}
$$

The solution of the problem is obtained through the use of the up-dated Lagrangian formulation of MCMEEKING and RICE (1975), this solution being the velocity field that minimizes

$$
\mathrm{I}=\frac{1}{2} \int_{\nu}\left[\frac{\mathscr{D} \tau^{i j}}{\mathscr{D} t} D_{i j}-\sigma^{i j}\left(2 D_{i k} D_{j l} g^{k l}-\left.\left.V^{k}\right|_{j} V_{k}\right|_{i}\right)\right] \mathrm{d} v-\int_{s} \dot{F}^{i} V_{i} \mathrm{~d} s .
$$

An appropriate generalization of the classical Prandtl-Reuss equations leads to the

\footnotetext{
$\dagger$ We follow Gurson (1977) in using the prefix "macro" to denote the average response of the cell and "micro" to denote pointwise variables within it.
} 


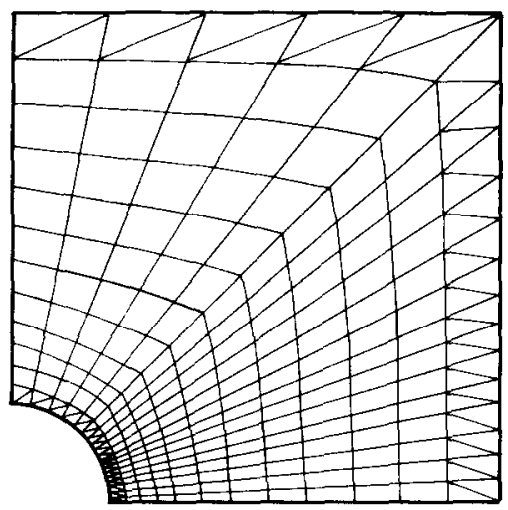

FIG. 2. The finite-element mesh.

following constitutive relations.

$$
\frac{\mathscr{D} \tau^{i j}}{\mathscr{D} t}=L^{i j k l} D_{k l}
$$

where

$$
L^{i j k l}=\frac{E}{(1+v)}\left[\frac{1}{2}\left(g^{i k} g^{j l}+g^{i l} g^{j k}\right)+\frac{v}{1-2 v} g^{i j} g^{k l}-\frac{3}{2 \sigma_{\mathrm{e}}^{2}} \frac{S^{i j} S^{k l}}{1+\frac{2(1+v)}{3 E} E_{t}^{(\mathrm{p})}}\right]
$$

for plastic loading (when $\sigma_{\mathrm{e}}=\sigma_{\mathrm{e}_{\max }}$ and $\mathscr{D} \sigma_{\mathrm{e}} / \mathscr{D} t>0$ ) and

$$
L^{i j k l}=\frac{E}{(1+v)}\left[\frac{1}{2}\left(g^{i k} g^{j l}+g^{i l} g^{j k}\right)+\frac{v}{1-2 v} g^{i j} g^{k l}\right]
$$

during elastic deformation or unloading from a plastic state (when $\sigma_{\mathrm{e}}<\sigma_{\mathrm{e}_{\max }}$ or $\mathscr{D} \sigma_{\mathrm{e}} / \mathscr{D} t<0$ ). We have replaced $E_{t}^{(\mathrm{p})}$ by $E_{t}$ and $\varepsilon_{\mathrm{e}}^{(\mathrm{p})}$ by $\varepsilon_{\mathrm{e}}$ in our analysis because the difference is negligible and it makes the computation easier.

Equations (2), (3) and the imposed boundary conditions were solved by the finiteelement method. The quadrant shown in Fig. 1 was subdivided into 456 constant strain triangular elements with 260 nodal points, $†$ as shown in Fig. 2. The development of the solution is most conveniently expressed by taking the elongation $W_{z}$ as the generalized time $t$. The boundary conditions used in the computations are

$$
\begin{array}{ll}
\dot{W}=\left(\Delta W_{z}\right) L_{0}, & \\
\dot{F}_{r}=0, & \text { at } z=L
\end{array}
$$

and

$$
\begin{aligned}
\dot{U} & =\left(\Delta U_{r}\right) R_{0}, \\
\dot{F}_{z} & =0, \quad \text { at } r=R .
\end{aligned}
$$

† This can be compared with NEEDLEMAN's (1972) plane strain analysis that used 198 nodes in a quadrant subdivided into constant strain elements. Comparison of the results of this mesh with those of a coarser one indicates that the mesh of 456 elements is fine enough to produce acceptably accurate results. 
The contraction of the cell is controlled by the value of $\alpha$ [defined by (1)] which ranges between zero and 0.5. $\alpha=0$ corresponds to the fixed lateral boundary used by ANDERSSON (1977), whilst $\alpha=0.5$ is approximately equivalent to incompressible behaviour of the embedding material.

In most cases the complete computation takes between 260 and 340 increments $\Delta t$ (i.e. $\Delta W_{z}$ ), the maximum loading being reached between 120 and 200 steps. The maximum increment used in the computations is chosen within the range $2 \times 10^{-4}-33$ $\times 10^{-4}$ according to the value chosen for $\alpha$. For small values of $\alpha$ one must choose a maximum increment that is towards the smaller end of this range. (This is because small $\alpha$ corresponds to larger dilatations and hydrostatic tensions which encourage rapid void growth and rapidly changing patterns of stress and deformation.) Because there is considerable change from elastic to elastic-plastic behaviour in many elements soon after first yield, the increments during the first 40 steps are varied from $\frac{1}{50}$ th of the maximum increment to the maximum increment itself at the 40th step.

The computation within each increment $\Delta t$ of time proceeds as follows.

(a) Specify the value of the increment $\Delta W_{z}$ of the normalized axial displacement of the cell.

(b) Within each element the tangent modulus to be used in the current load step is determined by the stress and strain conditions of that element at the end of the previous step. In particular, the inverse $E / E_{t}$ of the normalized tangent modulus is read from a predetermined Table $1 \dagger$ as a function of $\varepsilon_{\mathrm{c}}$. Linear interpolation is used for determining $E / E_{t}$ from this table.

(c) The functional I of (2) can now be evaluated by numerical integration. Ensuring that its variation, subject to the boundary conditions (4), is zero produces a matrix equation for the unknown nodal displacements and the inversion of the matrix yields the solution.

(d) In each element, the Jaumann derivative of Kirchhoff stress, the convected derivative of nominal stress and the change from nominal stress to true stress are calculated. The details have been given by $\mathrm{Lr}$ (1983). The Jaumann derivative of equivalent stress is also calculated in order to check whether the element is plastically loading or unloading.

(e) In each element the equivalent stress and strain are computed.

(f) Nodal points are updated and the process (a)-(f) is repeated for the next increment of time.

TABLE $1 . \varepsilon_{\mathrm{e}}-\left(E / E_{\imath}\right) \quad E=207 \mathrm{GPa}, v=0.29, \sigma_{\mathrm{Y}}=458 \mathrm{MPa}$

\begin{tabular}{ccccccc}
\hline$\varepsilon_{\mathrm{c}}$ & 0.010 & 0.030 & 0.040 & 0.050 & 0.065 & 0.080 \\
$E / E_{\mathrm{t}}$ & 68 & 76 & 90 & 120 & 142 & 154 \\
\hline$\varepsilon_{\mathrm{e}}$ & 0.100 & 0.120 & 0.140 & 0.500 & 0.800 & 1.10 \\
$E / E_{\mathrm{t}}$ & 236 & 340 & 440 & 450 & 460 & 470 \\
\hline & $\varepsilon_{\mathrm{e}}>1.10$ & $E / E_{\mathrm{t}}=470+40\left(\varepsilon_{\mathrm{e}}-1.10\right)$ & \\
\hline
\end{tabular}

$\dagger$ This is for material whose stress-strain record has been obtained $(\mathrm{Ll}, 1983)$ by simulating the material response during necking of a bar of $\mathrm{A} 533 \mathrm{~B}$ nuclear pressure vessel steel, and Table 1 represents these data. 
The convergence of the solution was checked for many typical cases by halving the increment of time taken throughout that computation until acceptable accuracy was achieved for the macro-response of the cell. For example, the results of Fig. 10 are converged to within $1 \%$ whilst those of Fig. 11 are within $5-7 \%$. This discrepancy is because the strains of Fig. 11 are an order of magnitude less than those of Fig. 10.

\section{Void Growth Without Softening}

The above procedures were used to analyse void growth in a hardening elasticplastic material whose stress-strain curve is represented in Table 1.

Computations have been performed for four model geometries in which

$$
r_{0}=0.05,0.10,0.20,0.40
$$

which correspond to initial void volume fractions of

$$
f_{0}=0.833 \times 10^{-4}, 0.666 \times 10^{-3}, 0.533 \times 10^{-2}, 0.426 \times 10^{-1} .
$$

For the cases of $r_{0}=0.05$ and 0.10 the mesh sizes around the void were smaller than those shown in Fig. 2, but the total number of nodes was the same.

The loading condition (1) gives an approximately proportional overall straining of the model cell. Deviations from proportional straining would occur if the loading displacement increment were too hig. Whenever the straining is proportional the following relationships are true.

$$
\begin{aligned}
\bar{\varepsilon}_{z} & =\ln \left(1+W_{z}\right), \\
\bar{\varepsilon}_{r} & =\ln \left(1+U_{r}\right)=-\alpha \bar{\varepsilon}_{z}\left[\text { since from }(1), \dot{\bar{\varepsilon}}_{r}=-\alpha \dot{\bar{\varepsilon}}_{z}\right] \\
\frac{\Delta R_{0}}{R_{0}} & =-U_{r}=1-\exp \left(-\alpha \bar{\varepsilon}_{z}\right), \\
\dot{\bar{\varepsilon}}_{\mathrm{e}} & =\frac{2}{3}\left(\dot{\bar{\varepsilon}}_{z}-\dot{\bar{\varepsilon}}_{r}\right)=\frac{2}{3}(1+\alpha) \dot{\dot{\varepsilon}_{z}}\left(\dot{\bar{\varepsilon}}_{\theta}=\dot{\bar{\varepsilon}}_{r}\right), \\
\bar{\varepsilon}_{\mathrm{e}} & =\frac{2}{3}\left(\bar{\varepsilon}_{z}-\bar{\varepsilon}_{r}\right)=\frac{2}{3}(1+\alpha) \bar{\varepsilon}_{z}\left(\bar{\varepsilon}_{\theta}=\bar{\varepsilon}_{r}\right), \\
\bar{\varepsilon}_{\mathrm{m}} & =\frac{2 \bar{\varepsilon}_{r}+\bar{\varepsilon}_{z}}{3}=\frac{1-2 \alpha}{3} \bar{\varepsilon}_{z}(\text { mean strain }), \\
\frac{\rho}{\rho_{0}} & =\frac{R_{0}^{2} L_{0}}{\left(R_{0}-\Delta R_{0}\right)^{2}\left(L_{0}+\Delta L_{0}\right)}=\frac{1}{\left[\exp \left(-\alpha \bar{\varepsilon}_{z}\right)\right]^{2}\left(1+W_{z}\right)} \text { or } \\
\ln \frac{\rho}{\rho_{0}} & =(2 \alpha-1) \bar{\varepsilon}_{z}=-3 \bar{\varepsilon}_{\mathrm{m}} .
\end{aligned}
$$

Define the average axial stress on plane $\mathrm{AB}$ in Fig. 1 as $\bar{\sigma}_{z}$ and the average radial stress on the cylindrical plane $\mathrm{BC}$ as $\bar{\sigma}_{r}$. Then

$$
\begin{aligned}
\bar{\sigma}_{\mathrm{e}} & =\bar{\sigma}_{z}-\bar{\sigma}_{r} \quad\left(\bar{\sigma}_{\theta}-\bar{\sigma}_{r}\right) \\
\bar{\sigma}_{\mathrm{m}} & =\frac{\bar{\sigma}_{z}+2 \bar{\sigma}_{r}}{3} \quad \text { (mean stress). }
\end{aligned}
$$




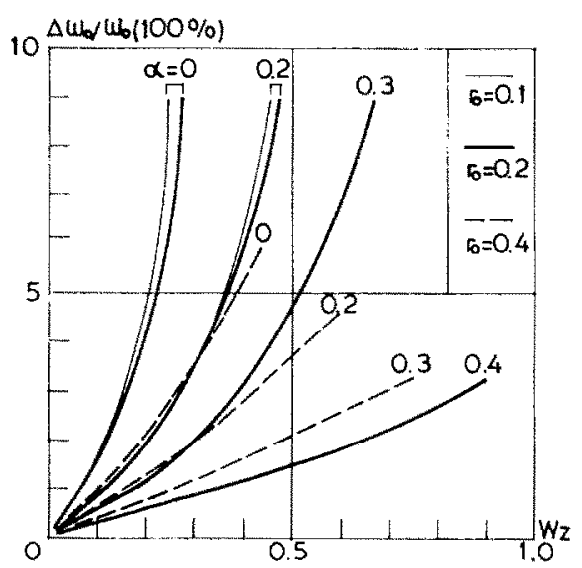

(a) STRAIN-HARDENING MODEL

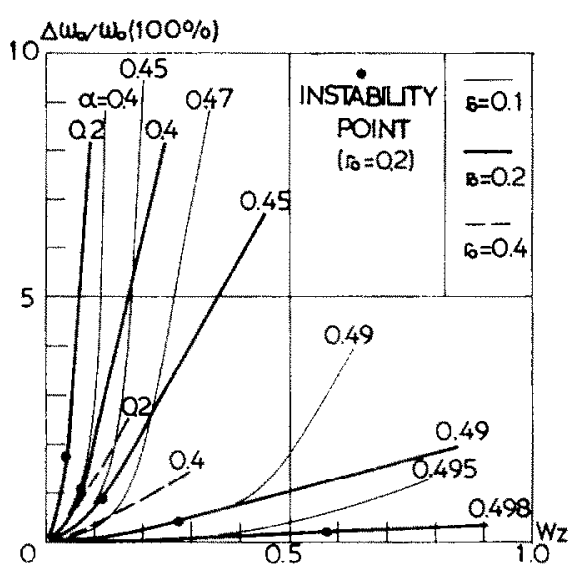

(b) STRAIN-SOFTENING MODEL

FIG. 3. The amount of void growth as a function of normalized elongation.

Computed results for the variation of void growth $\Delta \omega_{0}$ with the elongation $W_{z}$ are shown in Fig. 3(a). The results for $r_{0}=0.05$ and 0.10 coincide within the accuracy of the computations. The mean tensile stress $\bar{\sigma}_{\mathrm{m}}$ increases as the value of $\alpha$ decreases and the void grows faster. At the same clongation $W_{z}$, initially smaller voids have a greater growth rate. The exponential shape of the curves shown in Fig. 3(a) is similar to the experimental results of IRICIBAR, LE ROY and EMBURY (1980) and FISHER and GURLAND (1981) for spheroidized steels. Our results are also in qualitative agreement with the theoretical predictions of MCCunTock (1968). Figure 4 shows the size of the void as a function of equivalent strain and some results of MCCLINTOCK (1968) are also shown. Although the qualitative form of the results are the same, the fact that the ordinate is logarithmic means that the differences between the points on each curve are large. The spread of these values between different values of $\alpha$ exceeds, apparently, that for 


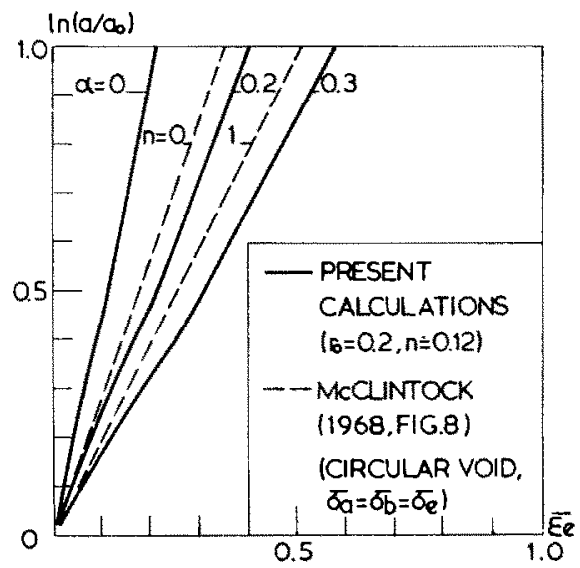

FlG. 4. The change of average void radius with equivalent strain.

differing hardening exponents. For purposes of comparison, the average hardening exponent of our material is 0.12 .

Figure 5 shows the relationship between $\bar{\sigma}_{\mathrm{m}}$ and $\bar{\varepsilon}_{\mathrm{m}}$ for various void volume fractions and degrees of dilatation. High values of the maximum mean stress are associated with small initial void sizes and/or high dilatation, and the results shown all have the peak in $\bar{\sigma}_{\mathrm{m}}$ in excess of $7 \%$ of $E$. Furthermore, the local values of stress and strain within the cell can exceed even these. But stresses of the order of $E / 10$ exceed the theoretical tensile strength of most materials. Also, it is commonly believed that steels in particular would already have initiated second order voids at the sites of fine carbides and other precipitates at these huge stresses (see HANCOCK et al, 1976, 1980; LAUTRIDOU and PINEAU, 1981). We are convinced by these facts that the model of monotonic hardening often used to discuss metal plasticity is entirely inappropriate after some (materially dependent) level of stress/strain is reached in the matrix. The simplest remedy is to assume that the matrix softens.

\section{VoId Growth with SOFTENING}

The BEREMIN group (1981b) have suggested that voids nucleate around inclusions when the stress reaches a critical value

$$
\sigma_{\mathrm{c}}=\sigma_{\mathrm{m}}+\left(\frac{2}{3}+\lambda\right) \sigma_{\mathrm{e}}
$$

The value of the parameter $\lambda$ is dependent on the shape of the inclusion and, for typical shapes, its value lies between 0.4 and 1.7 with a value of one for spherical voids. The second term in (5) is concerned with the role of equivalent strain and so the formula can be regarded as the relationship between mean stress and equivalent strain that must exist as the void nucleates; $\sigma_{\mathrm{c}}$ and $\lambda$ are material constants.

Figure 6 shows some of the predictions of $\operatorname{LI}(1983)$ for the distribution of stress in the neck of a tensile round bar, and it can be seen that, just before fracture of the bar, the 


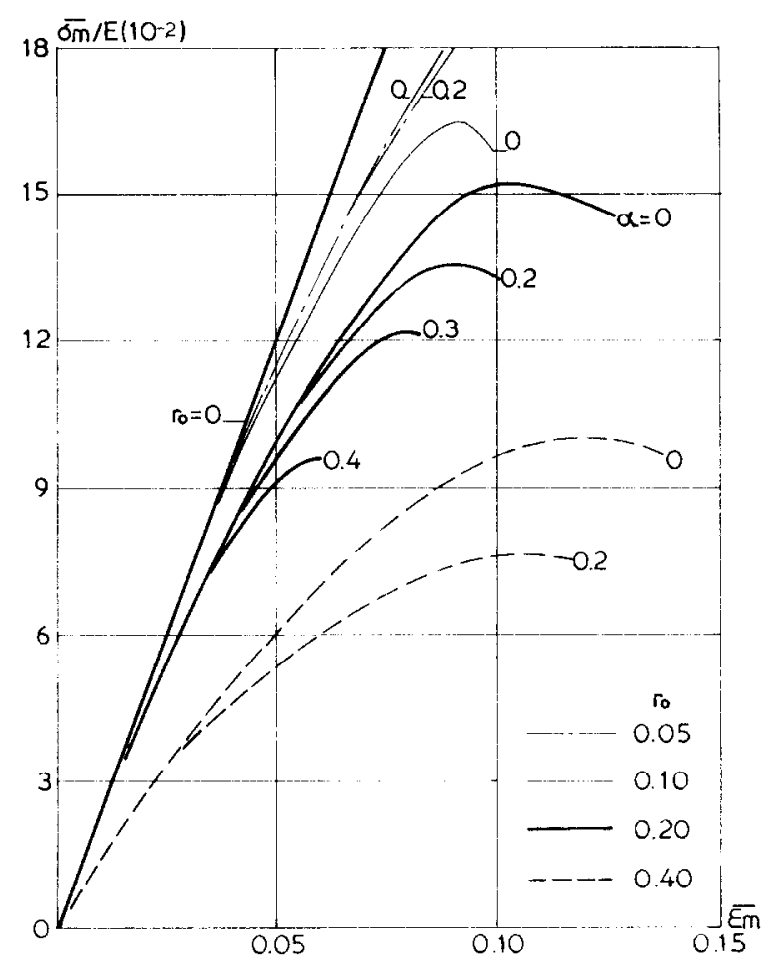

Fia. 5. Computed values of mean tensile stress and Intean strain for void growth in it matrix that does not soften.

centre of the neck deforms so that

$$
\sigma_{\mathrm{e}}=\sigma_{z}-\sigma_{r}=2.5 \sigma_{\mathrm{Y}}, \quad \sigma_{\mathrm{m}}=-\frac{\sigma_{z}+2 \sigma_{r}}{3}=1.71 \sigma_{\mathrm{Y}}
$$

Equation (5) (with $\lambda=1$ ) then predicts $\sigma_{\mathrm{c}}$ will have been reached before the value

$$
\sigma_{\mathrm{c}_{\max }}=5.89 \sigma_{\mathrm{Y}}
$$

In the following calculations, it is assumed that $\sigma_{\mathrm{c}} / \sigma_{\mathrm{Y}}=4$.

A simple way of taking softening into account is to assume as in Fig. 7 that the equivalent stress-strain curve has a softening branch $\mathrm{AD}$ besides the plastic loading path $\mathrm{AB}$ and the elastic unloading branch $\mathrm{AC}$. The main difference between $\mathrm{AD}$ and $A C$ is that passage along the softening path is irreversible. This kind of material response is in accord with the experiments of MACKENZIE, HANCOCK and BROWN (1977) who showed that there was a sharp drop in the loading stress after the initiation of macro-fracture in the tension tests.

[The connection between softening and the load drop observed by Mackenzic, Hancock and Brown is somewhat subtle, in that our computer simulations (not reported here) of their experiments are unable to predict the load drop correctly without the incorporation of material softening. The point of material softening and that of the load drop are not the same on any scale of deformation or load chosen to 


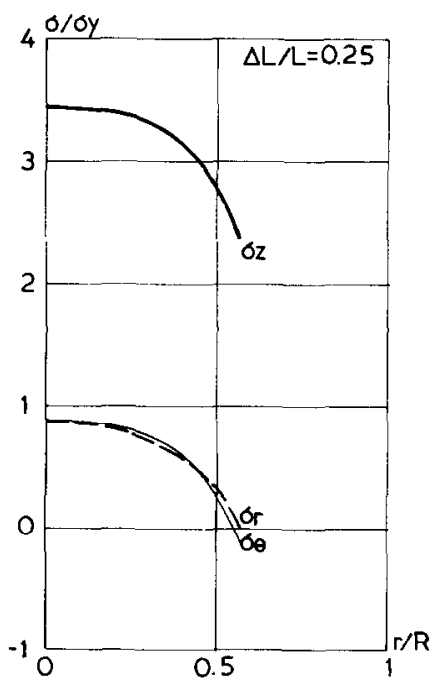

FIG. 6. The distribution of stress in the mid-plane of a round necking bar at $\Delta L / L=0.25 . R$ is the initial maximum radius of the bar and $L$ is the initial half length of the bar.

measure the tensile test, but they will usually be close when measured by the global elongation.]

We now assume that at any point $\mathrm{A}$ on the loading path where the critical criterion (5) is met, irreversible strain-softening occurs. Mathematically, if

$$
\sigma_{\mathrm{m}}+\left(\frac{2}{3}+\lambda\right) \sigma_{\mathrm{e}}>\sigma_{\mathrm{c}}
$$

then the positive tangent modulus in the constitutive equation (3) is replaced by a softening modulus $E_{\mathrm{f}}$ with negative sign, i.e.

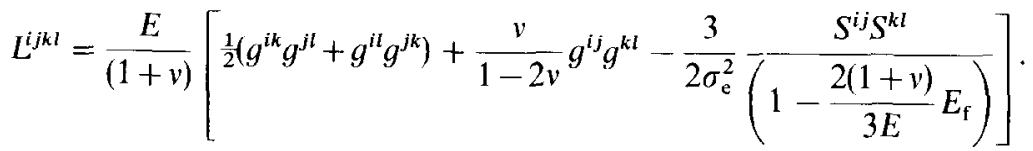

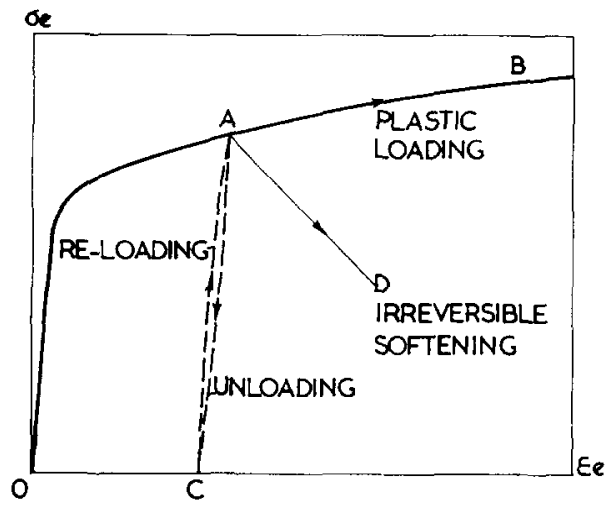

FIG. 7. Schematic equivalent stress/equivalent strain curve, showing unloading and softening branches. 


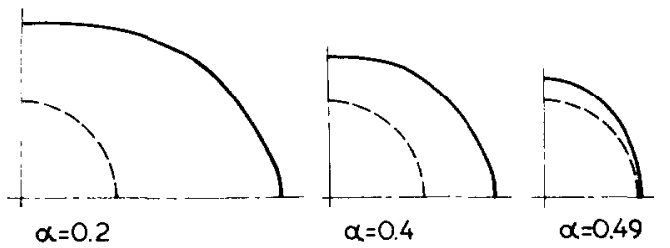

$-\cdots$ INITLAL VOID(ro=0.2)

\section{- CURRENT SIZE(AT WZ $=0.113$ )}

FlG. 8. Void shapes at $W_{z}=0.113\left(r_{0}=0.20\right)$.

In the following calculations the normalized inverse $F / F_{\mathrm{f}}$ of the softening modulus is taken to be 100 .

Our earlier computations show that there is no need to consider the case $r_{0}=0.05$ since the overall response for that case coincides with that for $r_{0}=0.10$ because the interaction with neighbouring voids is negligible for small initial void sizes.

Figure 3(b) shows the void growth $\Delta \omega_{0}$ as a function of the normalized elongation $W_{z}$. The void growth rate (for the same value of $\alpha$ ) is larger than the corresponding one in Fig. 3(a) because the matrix is much softer. The shape to which the void grows depends on the value of the parameter $\alpha$, and its effect is illustrated in Fig. 8. Generally, the void grows radially when $\alpha<0.4$ (i.e. in the case of high mean stress) and axially otherwise.

The macroscopic axial and radial stresses for $r_{0}=0.20$ are shown in Fig. 9. When $\alpha<0.49$ the points of instability are very definite and $\bar{\sigma}_{z}$ and $\bar{\sigma}_{r}$ reach a limit point at nearly the same generalized time. For $\alpha>0.49$, however, the pattern is quite different.

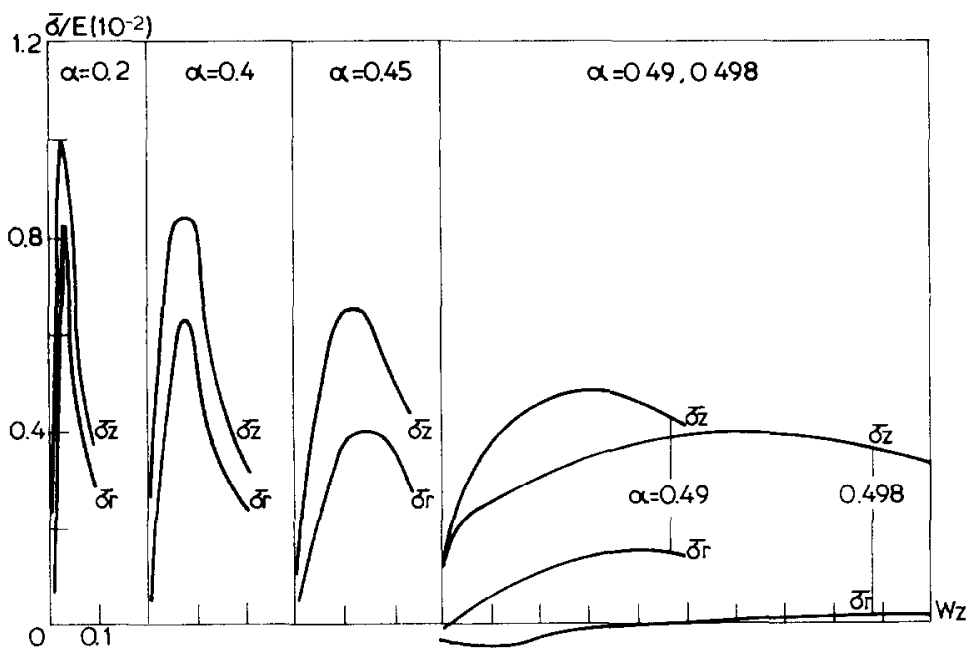

Fig. 9. Computed values of the macroscopic cell stresses as a function of elongation $\left(r_{0}=0.20\right)$. 


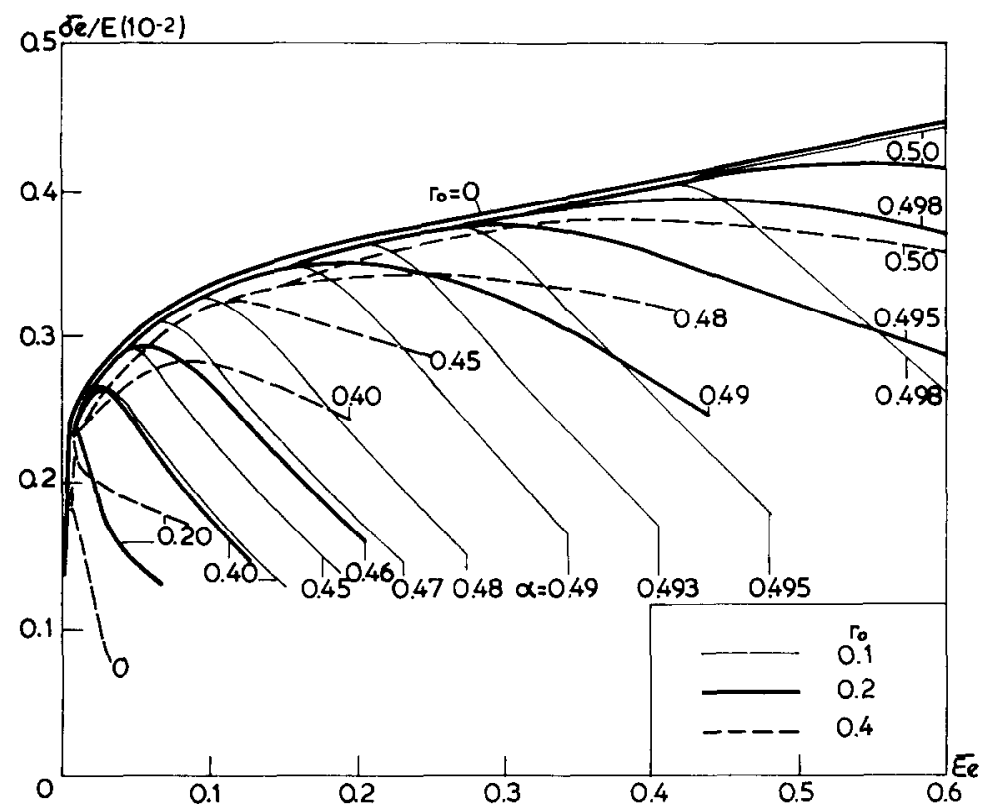

Fig. 10. Equivalent stress-strain curve of a cell with a matrix that softens.

The limit point of $\bar{\sigma}_{r}$ occurs later than that for $\bar{\sigma}_{z}$ and even becomes compressive if $\alpha$ is very close to 0.5 . The effect on the axial stress is merely to flatten the curve.

These stresses $\bar{\sigma}_{z}$ and $\bar{\sigma}_{r}$ may be decomposed into the equivalent stress $\bar{\sigma}_{\mathrm{e}}$ and mean stress $\bar{\sigma}_{\mathrm{m}}$ and their variation with $\bar{\varepsilon}_{\mathrm{m}}$ and $\bar{\varepsilon}_{\mathrm{e}}$ for different $r_{0}$ and $\alpha$ are shown in Fig. 10 and Fig. 11. The corresponding limiting case of $r_{0}=0$ is also shown in solid lines. Softening of the matrix yields, as expected, enhanced softening of the $\bar{\sigma}_{\mathrm{e}} / E-\bar{\varepsilon}_{\mathrm{e}}$ and $\bar{\sigma}_{\mathrm{m}} / E-\bar{\varepsilon}_{\mathrm{m}}$ curves. This can be seen by comparing the results in Fig. 11 with those of Fig. 5. Figure 10 shows the $\bar{\sigma}_{\mathrm{e}}-\bar{\varepsilon}_{\mathrm{e}}$ curves for different void volume fractions and cell dilatations. For the same value of $r_{0}$ the loading part of each of these curves are coincident, and the unloading parts have approximately the same slope. These observations also apply to Fig. 11, which shows corresponding $\bar{\sigma}_{\mathrm{m}}-\bar{\varepsilon}_{\mathrm{m}}$ curves, but in a way which is less precise. This suggests that the behaviour of real ductile materials can be simulated on a computer by simple approximations to the shapes shown in Figs 10 and 11. The results of this work will be reported in another paper.

Figure 12 shows the variation of the triaxiality parameter $\bar{\sigma}_{\mathrm{m}} / \bar{\sigma}_{\mathrm{e}}$ with $\bar{\varepsilon}_{\mathrm{e}}$ at the point of instability, taking this to be the limit point on the $\bar{\sigma}_{z}-W_{z}$ curve shown in Fig. 9. For the same value of $r_{0}$, high values of $\bar{\sigma}_{\mathrm{m}} / \bar{\sigma}_{\mathrm{e}}$ at instability correspond to small values of $\bar{\varepsilon}_{\mathrm{e}}$ and $\alpha$, and the value of $\bar{\sigma}_{\mathrm{m}} / \bar{\sigma}_{\mathrm{e}}$ drops exponentially with increasing $\bar{\varepsilon}_{\mathrm{e}}$. Larger values of $r_{0}$ depress the whole curve bodily towards the coordinate axes. These results are in qualitative agreement with the analyses of ROUSSELIER (1981) and the experimental work of HANCOCK and MACKENZIE (1976), HANCOCK and Cowling (1980) and LaUtridou and Pineau (1981). For $r_{0}=0.10$ and $\alpha>0.48$ the $\bar{\sigma}_{z}-W_{z}$ relationship is 


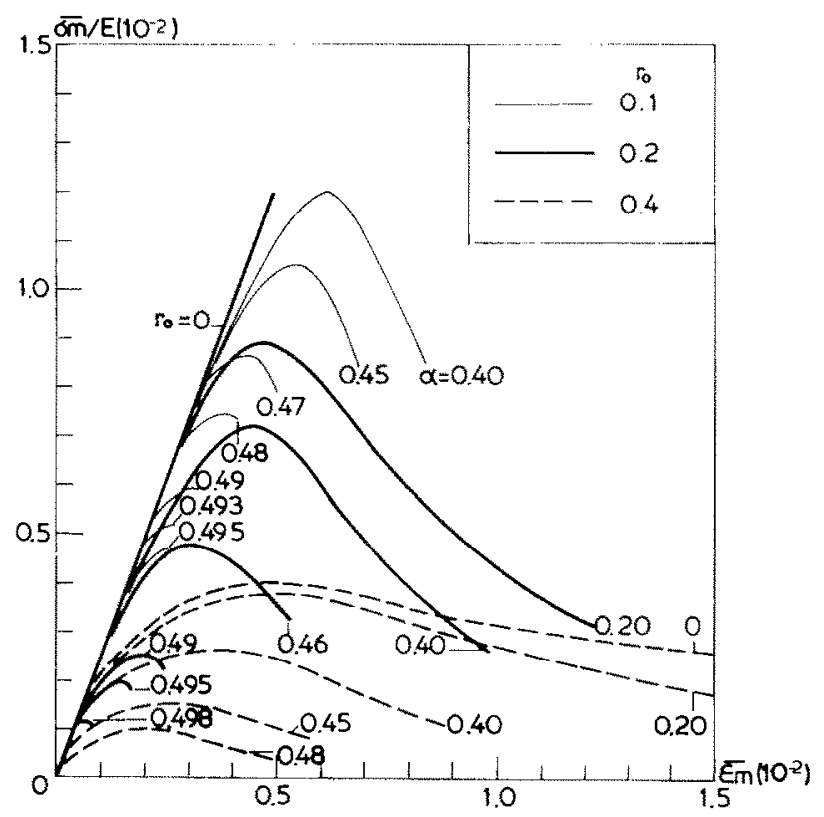

Fig. 11. Mean stress-strain curve of a cell with a matrix that softens.

very flat, the original limit point widens to a quasi-plateau and there is no practically obvious peak point to be taken as the occurrence of instability.

In Fig. 13 is shown the relationship between the void growth parameter $\dot{\omega} / \omega \dot{\overline{\mathrm{e}}}$ and a normalized mean stress $\bar{\sigma}_{\mathrm{m}} / \sigma_{\mathrm{\gamma}}$. The curves are terminated at the instability point. The results of the work of RICE and TRACEY (1969) are re-plotted by turning their $\dot{a} / a$ into

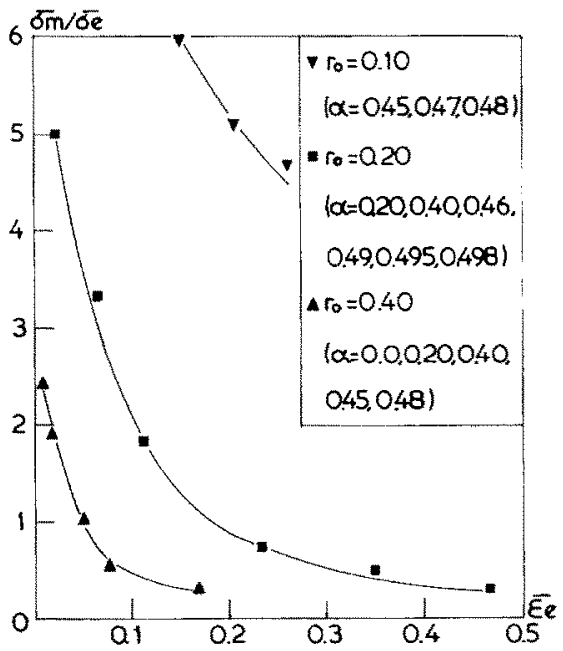

Fis. 12. The triaxiality parameter $\bar{\sigma}_{\mathrm{m}} / \bar{\sigma}_{\mathrm{c}}$ vs equivalent strain at the instability point. 


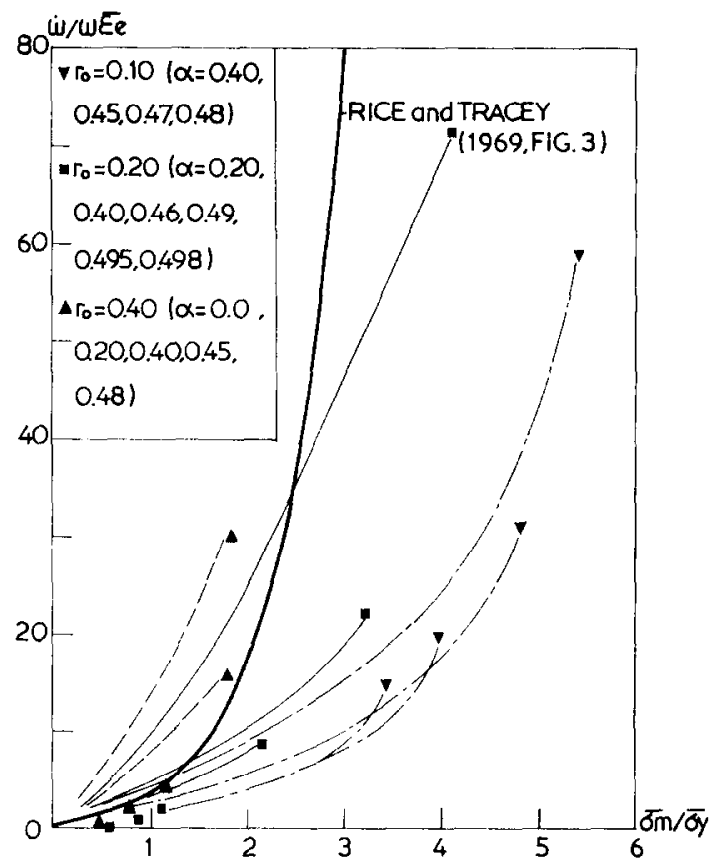

FIG. 13. The void growth parameter $\dot{\omega} / \omega \hat{\varepsilon}_{\mathrm{v}}$ vs normalized mean stress (the curves have been terminated at the instability point).

$\dot{\omega} / \omega$ since

$$
\frac{\dot{\omega}}{\omega}=\frac{3 \dot{a}}{a}
$$

approximately for small increments. Rice and Tracey analysed the growth of a spherical void embedded in an infinite perfectly plastic medium. It is of some interest to note that our results for $r_{0}=0.10$ predict a higher tensile stress than theirs even though our material softened after the attainment of the critical stress $\sigma_{\mathrm{c} .}$. This is probably due to the matrix hardening in our computations that exists before $\sigma_{\mathrm{c}}$ is reached. Both Figs 4 and 13 make comparison between the present computations and results which have been extensively used to analyse ductile fracture. An important point to emerge is that the spread of our results is large and may even involve an order of magnitude of difference. If the real processes being analysed depend strongly on these variables the choice of the inappropriate curve will introduce significant errors of interpretation.

The initiation of strain-softening in any of the constant strain finite elements was recorded during the computation. Three stages of this initiation are recorded in Fig. 14 where $W_{\mathrm{m}}$ denotes the value of the elongation $W_{z}$ at the instability point. For $r_{0}=0.10$ and $\alpha=0.498$ the determination of $W_{\mathrm{m}}$ suffered from the errors already discussed (i.e. the limit point on the $\bar{\sigma}_{z}-W$, curve widens to a quasi-plateau) so an approximate value of $W_{\mathrm{m}}$ is chosen. The pattern of softening depends strongly on $r_{0}$. When $r_{0}$ is small and $\alpha<0.40$ most of the matrix material has softened in the first stage of elongation. This early softening is suppressed as the value of $\alpha$ or $r_{0}$ increases. It then begins 

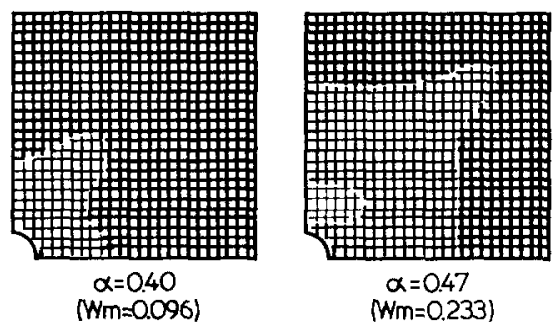

$\alpha=0.47$

$\mathrm{Nm}=0.233)$

(a) $r_{0}=0.1$

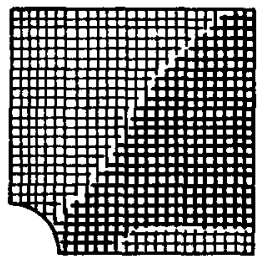

$\alpha=0.20$

$(W m=0.042)$

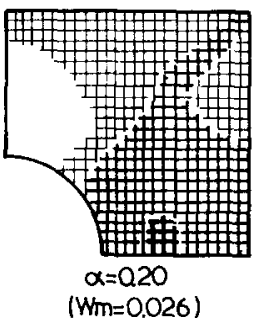

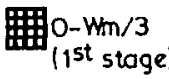

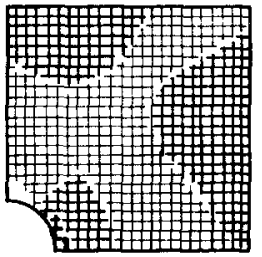

$\alpha=0.46$

( $W \mathrm{~m}=0.114$ )

(b) $r_{0}=0.2$

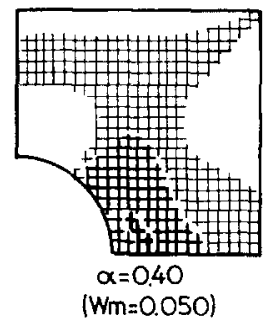

(c) $50=0.4$

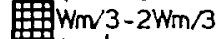

(2nd stage)

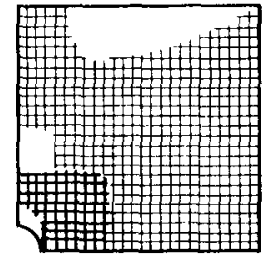

$\alpha=0.498$

( $W m=0.513$ )
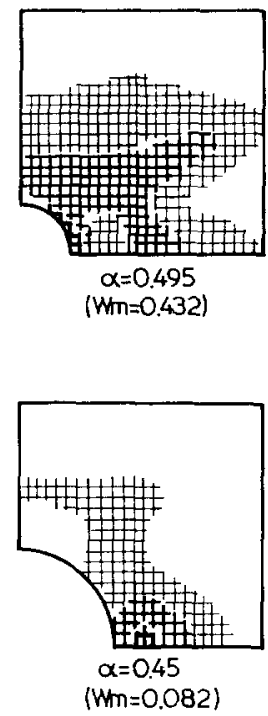

${ }_{\left(3^{\text {rd }} \text { stage }\right)}^{2 W / 3-W m}$

FIG. 14. The initiation and development of strain softening in the matrix.

later in generalized time and tends to localize, leaving a greater proportion of the matrix still hardening at the instability point.

These results throw some light on the mechanical and geometrical variables that may control the way in which the material fails during ductile fracture. When $r_{0}$ is small or if there is a high mean tensile stress (when $\alpha$ is small), the void growth rate is high since the void is surrounded by softening material. Fracture by void coalescence is therefore probable in this regime controlled by a critical void size or growth rate. On the other hand, with larger $r_{0}$ or small mean tensile stress (when $\alpha$ is larger), the softening is localized and this would favour a failure by shear decohesion.

Our computations show that the ability of the material to soften after some critical point has been reached may have a profound effect on the macroscopic behaviour of a cell consisting of a void growing in the matrix. The fact that the softening of the material enhances that of the cell does not, of course, need to be demonstrated by expensive 
computations, but the extent of it does and requires some comment. The maximum achievable stress $\left(\bar{\sigma}_{\mathrm{m}}\right)$ on the cell is reduced by an order of magnitude when the matrix is allowed to soften in the way that we suggest, and this is a more realistic prediction for many engineering materials than that associated with indefinite hardening. Furthermore, the elongation to achieve a given increase of size of the void is reduced by a factor of about 3-5 when softening occurs in the matrix. Apart from the values chosen for $\sigma_{\mathrm{c}}$ and $\lambda$ these estimates for the change between hardening and softening behaviour depend on that chosen for the softening modulus $E_{\mathrm{f}}$. Comparison of Fig. 12 with the experimental results of HANCOCK and MACKENZIE (1976) and LAUTRIDOU and PINEAU (1981) shows that our choice may be reasonable at least for steels.

Figure 12 can be regarded as a theoretical prediction of the kind of $\bar{\sigma}_{\mathrm{m}} / \bar{\sigma}_{\mathrm{c}}-\bar{\varepsilon}_{\mathrm{e}}$ damage function that is sometimes used in discussing ductile fracture. It clearly shows the influence of initial void volume fraction, and there is a hidden dependence on the characteristics of the matrix material (the computations reported here are for a material that hardens like A533 B nuclear pressure vessel steel and softens after $\sigma_{\mathrm{c}}=4 \sigma_{\mathrm{Y}}$ with a modulus of $E / 100$ ). This would now allow information about the size of the particles around which the voids nucleate to predict a definite damage function which could then be used to predict the ductile fracture characteristics of the material.

Although we have taken ductile failure to occur at the point of instability of the cell the damage function could be controlled by other events which either precede or follow it. For example, the kind of bifurcation that occurs with the localization of flow within the matrix may be the controlling event in the formation of the damage function. This can readily be seen by examining the instability points of Fig. 3(b) which would have been preceded by some event of failure in the hardening matrix. There is a little evidence to support the hypothesis that, if the mechanical behaviour of the matrix material is correctly represented, the points of instability and of bifurcation (due to localization and shear decohesion) would be indistinguishable at least in load if not elongation. Our computations are, of course, tentative, and the particular softening we have included is based to a certain extent on guesswork. Further computation and, more importantly, comparison with experimenlal data is needed before these and other questions can be resolved. These observations make some connection between two of the different criteria of ductile fracture mentioned in the Introduction. The other, the attainment of a critical size of the void, is less likely than the other two to be a universally valid criterion, at least on the evidence of our computations. Figure 3(b) shows that, for a given void volume fraction, the size of the void at which instability occurs drops exponentially with the elongation. For values of $\alpha$ smaller than about 0.4 the point of instability can be taken at roughly the same size because the rate of void growth with elongation is very high and a void doubles its size during a very small increasc in clongation. This suggests that the concept of failure at a critical size of void is likely to be confined to cases of moderate to high triaxiality. Our results have been obtained with one set of values $\left(\lambda=1, \sigma_{\mathrm{c}}=4 \sigma_{y}, E_{\mathrm{f}}=E / 100\right)$ of the parameters which control the softening of the matrix. Furthermore, different values of these will interact differently with the other mechanical and geometrical parameters that control the deformation of the cell. Our understanding of the effects of softening will be considerably enhanced when we know the effects of changing these parameters, and this will be the subject of a subsequent paper. 
ANDERSSON, $\mathrm{H}$.

BerEMin, F. M.

FISHER, J. R. and Gurland, J.

GuRSON, A. L.

HANCOCK, J. W. and Brown, D. K.

HANCOCK, J. W. and Cowling, M. J.

HANCOCK, J. W. and MACKENZIE, A. C.

IRICIBAR, R., LE ROY, G. and EMBURY, J.D.

LAUTRIDOU, J. C. and Pineau, A.

LI, G. C.

Mackenzil, A. C., Hancock, J. W. 1977 and Brown, D. K.

McClintock, F. A.

MCMEEKING, R. M. and RICE, J. R.

Needleman, A.

NorRIS, D. M., Reaugh, Jr. J. E., MORAN, B. and QUINONES, D. M.

Rice, J. R. and Tracey, D. M. ROUSSELIER, G.

\section{RFFERENCES}

1977 J. Mech. Phys. Solids 25, 217.

1981a Advances in Fracture Research (edited by D. Francois), ICF5, Cannes, France, 29 March3 April 1981, Vol. 2, p. 809. Pergamon Press, Oxford.

1981b Met. Trans. 12A, 723.

1981 Metal Sci. 15, 185.

1977 J. Eng. Mat. Technol. 99, 2.

1983 J. Mech. Phys. Solids 31, 1.

1980 Metal Sci. 14, 293

1976 J. Mech. Phys. Solids 24, 147.

1980 Metal Sci. 14, 337.

1981 Engng. Fracture Mech. 15, 55.

1983 Int. J. mech. Sci. 25 (to be published).

Engng. Fracture Mech. 9, 167.

1968 J.appl. Mech. 35, 363.

1975 Int. J. Solids Struct. 11, 601.

1972 J.appl. Mech. 39, 964.

1978 J. Eng. Mat. Technol. 100, 279.

1969 J. Mech. Phys. Solids 17, 201.

1981 Advances in Fracture Research (edited by D. Francols), ICF5, Cannes, France, 29 March3 April 1981, Vol. 2, p. 803. Pergamon Press, Oxford. 